Andrea Schembari

Istituto di Letteratura e Nuovi Media, Università di Stettino

\title{
Maestri del dissenso: Leonardo Sciascia e la lezione degli scrittori polacchi
}

\section{Dissentire e/è testimoniare}

Fin dalla pubblicazione del fondamentale studio di Ricciarda Ricorda sulla "retorica della citazione" [Ricorda 1977], la trama intertestuale della scrittura di Leonardo Sciascia ha offerto agli studiosi un fecondo campo di indagine, e ha favorito in modo particolare lo sviluppo di una bibliografia critica dedicata alle intersezioni di quella scrittura con la letteratura europea di ogni tempo. Se il lavoro di scavo è stato inizialmente rivolto soprattutto agli assi portanti della letteratura francese [cfr. Della Terza 1990; Simonetta 1996; Bosco 1999; Morrison 2009] e spagnola [Tedesco 1989 e 1991, Manganaro 1990, Gioviale 2000], più di recente l'attenzione si è spostata su culture e tradizioni letterarie di altre regioni, dalla Svizzera [cfr. Martinoni 2011] alla Jugoslavia [cfr. Ricorda 2015], dalle aree di lingua tedesca [cfr. Fontana, Pupo 2019] agli scrittori russi [cfr. Nocera 2020].

Come per tempo segnalato [cfr. Ferraro 1993 e Kazana 1997] la mappa storica e letteraria d'Europa su cui Sciascia si è orientato per la propria scrittura, ha incluso anche la Polonia e i suoi autori, cui lo scrittore - si può già affermare - ha fatto ricorso come a un paradigma espressivo del dissenso politico, culturale, 
o etico. Prima di avviare una ricognizione dei loci sciasciani su cui verificare l'assunto, può essere però utile ricordare un episodio. $\mathrm{Nel}$ marzo del 1964 Leonardo Sciascia visitò a Roma, presso la Galleria dell'Obelisco di Irene Brin e Gaspero Del Corso, la mostra del pittore polacco Marian Warzecha. Negli stessi giorni Laterza pubblicava Morte dell'inquisitore, il saggio di inchiesta storica che lo scrittore dedicò al frate Diego La Matina, suo conterraneo, arso vivo dal tribunale della Santa Inquisizione nel 1658. La coincidenza suggerì a Sciascia l'incipit di una nota per il quotidiano "L'Ora" di Palermo:

C'erano molte signore e letterati e pittori: ma di colpo, tra i quadri del Warzecha, io mi sono sentito come doveva sentirsi il vecchio Pitré tra le mura del carcere del Palazzo Chiaramonte, nei mesi che vi passò a decifrare le scritte dei prigionieri. Perché il mondo di Warzecha [...] era proprio quello su cui Pitrè si era chinato attento e pietoso: il mondo del carcere, il mondo dell'inquisizione [... ]. Benché nella presentazione del catalogo non se ne facesse menzione, non c'era dubbio che i quadri di Warzecha ossessivamente ripetessero i graffiti e le scritte di un carcere. I dati di una particolare esperienza venivano così portati fuori, di fronte a coloro che si credevano liberi: e assurgevano a significato di una condizione ancora, e per tutti, attuale. Un avviso, un ammonimento [Sciascia 1991: 6].

Non sono molte le notizie biografiche disponibili su Marian Warzecha, pittore e scenografo nato a Cracovia nel 1930, che esordì giovanissimo dedicandosi sin dagli inizi alla tecnica del collage, sviluppata con l'utilizzo di supporti cartacei tratti da vecchi volumi e stampe, di forme e colori disposti secondo rigorosi rapporti di proporzione e di evidente effetto geometrico. In una intervista del 2018 per il Muzeum Sztuki Współczesnej di Cracovia, l’artista non fa particolari accenni a una eventuale e sofferta condizione di controllo da parte delle autorità; ${ }^{1}$ e in effetti, il pieghevole della 
presentazione della mostra non accenna ad alcuna dimensione carceraria o inquisitoriale nella produzione dell'artista. Ma un passaggio del breve testo (tra altri - a dire il vero - piuttosto concettosi e allusivi) esplicita due elementi che devono avere suggerito quella analogia allo scrittore:

Warzecha ci presenta questi suoi diari di meditazione che $[. .$. posseggono tutti gli attributi di una vera avanguardia e di una piena coscienza [... . Mi sembra che Warzecha parta da un problema filosofico a priori come testimonianza della esistenza che lui cerca, scegliendo come bersaglio delle sue ricerche un invincibile e patetico specchio - il tempo [...]. Sembra quasi che Warzecha si opponga e si vendichi dell' inesorabile, legando con gesto inventivo e superbo il tempo; lo irrazionalizza e lo concentra evocando i documenti del passato, lasciando l'impronta del segno dell'attualità [Drago 1964].

Manipolare e comporre i lacerti dei documenti del passato, secondo forme e colori regolari e armonici, serviva a sterilizzare l'effetto dissipatore e mistificatore del tempo, a sottoporlo a una nemesi necessaria e continua, cui l'artista chiamava a partecipare il pubblico; un approccio ermeneutico ed espressivo non dissimile da quello operato da Sciascia, specie in quegli anni Sessanta, per cui la necessità di una scrittura che sia anche nemesi delle storture del tempo, del passato, passava per il riutilizzo dei documenti della Storia, e assumeva la forma di un manifesto etico:

Tutto è legato, per me, al problema della giustizia: in cui si involge quello della libertà, della dignità umana, del rispetto tra uomo e uomo. Un problema che si assomma nella scrittura, che nella scrittura trova strazio o riscatto. E direi che il documento

e incoraggiamento per la scelta di specializzarsi nella tecnica del collage, per il timore dei docenti di attirare l'attenzione delle autorità; in un altro parla delle sue prime mostre, alludendo con molta noncuranza al fatto che sì, i rappresentanti del partito presenziavano perché dovevano, ma non avevano granché da indagare: anche perché nulla comprendevano [cfr. Warzecha 2018]. 
mi affascina - scrittura dello strazio - in quanto entità nella scrittura, nella mia scrittura, riscattabile [Sciascia 1987: XIII].

A quell'altezza, le vicende storiche e gli autori della Polonia "eroica e sventurata" [Sciascia 2012: 96] avevano tuttavia già tramato La morte di Stalin, il racconto incentrato sulla figura del ciabattino Calogero Schirò, che vive nella venerazione di Stalin e attraversa tra angustie, delusioni e illusioni - le contraddittorie vicende storiche che vanno lentamente demolendo l'immagine del suo idolo: dal patto Molotov-Ribbentrop del 1939 alla divulgazione del rapporto di Nikita Chruščëv, nel 1956, che diede avvio al processo di destalinizzazione in Unione Sovietica e nei paesi satelliti.

La riscrittura mentale e quotidiana della propria mitografia di Stalin, a controcanto della scoraggiante cronaca quotidiana, induce il protagonista a costruirsi una propria verità, fatta di ipotesi e supposizioni sulle future (e sempre imminenti) mosse decisive del suo eroe: che, dal canto suo, interviene personalmente a rincuorarlo, a dargli la giusta spiegazione e interpretazione dei fatti e delle vicende politiche che tanto lo affannano: "[...] non era la prima volta che vedeva Stalin in sogno, e dai fatti era poi risultata la verità del sogno. Niente di soprannaturale, si capisce: Stalin pensava e in sogno lui riceveva quel pensiero, anche gli scienziati lo ammettono" [Sciascia 2012: 94]. La cruda realtà tormenta però Calogero, incarnata dai ragionamenti che gli oppone, durante le conversazioni che si tengono nella sua bottega, l'arciprete del paese. Ed è qui che le tristi e note vicende polacche, di quel 1939, si materializzano nella pagina di Sciascia:

Intanto Hitler si mangiava la Polonia, il suo esercito si muoveva come uno schiaccianoci, la Polonia di colpo frantumata, la Polonia marcia di latifondismo, pensava Calogero, l'eroico popolo polacco, quei marci latifondisti che guidavano cariche di cavalleria contro i carri armati di Hitler, tutta la Polonia con un solo grande cuore, viva la Polonia eroica e sventurata. Gli veniva voglia di mettersi a gridare in piazza "viva la Polonia" e piangeva leggendo le corrispondenze di guerra, anche i giornalisti fascisti parevano commossi quando scrivevano della 
Polonia che moriva, uno di loro scrisse sulla caduta di Varsavia un pezzo che Calogero ritagliò dal giornale e conservò nel portafoglio. Quando la Russia si mosse a prendere la sua parte di Polonia l'arciprete si rifece vivo, si appoggiò alla porta e disse "tu dovresti saperlo l'inno di Mameli" e Calogero non capì dove volesse andare a parare, lo sapeva l'inno di Mameli, non lo ricordava tutto ma ce l'aveva in un libro. E l'arciprete disse "leggilo, dove dice 'il sangue polacco bevé col cosacco' mettici un bel pensierino sopra, quello che la coscienza ti detta". [Sciascia 2012: 96].

Non si deve fare troppo sforzo per riportare il sentimento del calzolaio a quello dello scrittore, che paragonava il dolore provato per l'accordo tra la Germania nazista el'Unione Sovietica a quello della perdita del fratello Giuseppe: ${ }^{2}$ e sembra di vederlo, il giovane studente Sciascia, che ritaglia quel reportage e lo custodisce con cura nel portafoglio. A differenza del suo personaggio d'invenzione, tuttavia, per lo scrittore quel momento fu l'inizio di un percorso di liberazione, di un affrancamento dalle costrizioni ideologiche di partito: una scelta di indipendenza che contraddistinguerà la sua vita intellettuale e politica.

Anche il tormentato e tetragono sistema di auto-convincimento di Calogero sembra vacillare alle notizie che giungono dal fronte di guerra, senza che questo faccia però mai sorgere il dubbio provvidenziale, la benefica contraddizione che conduca a un ripensamento delle proprie posizioni. Nemmeno quando l'autore presta al suo personaggio le parole della grande letteratura, che potrebbero condurlo a dissentire innanzitutto da se stesso. Alla morte di Stalin, nel pieno dello smarrimento personale e politico, Calogero sente infatti immediata la necessità di trovare una giustificazione anche storica alla figura del suo mito, cercando appiglio nella sua modesta (ma non mediocre) cultura, e svolgendo un ragionamento che mescola fede e giustizia sociale: 
Il giudizio della storia, ora. Ma Stalin era la storia stessa. Il giudizio di Dio. Ammettiamo che ci sia Dio, che tenga il libro nero e il libro bianco, che abbia in mano la bilancia della giustizia. E Stalin che cosa ha dato se non giustizia? E agli uomini cui non poteva giungere a dare giustizia non dava forse speranza? Fede speranza carità. No, niente carità: fede e speranza. E giustizia. Aveva spremuto dolore dagli uomini Stalin; aveva camminato col passo della rivoluzione, il passo della violenza e del sangue; ma una rivoluzione deve essere rivoluzione, Cristo che era Cristo portava una parola nuova che grondava sangue, Calogero aveva letto il Quo vadis?, quella gente non ammazzava ma si faceva ammazzare, ed era la stessa cosa [Sciascia 2012: 113].

A riprova della necessaria e cruenta violenza insita in ogni rivoluzione, Calogero Schirò richiama dunque l'esempio dello sconvolgimento portato nella Storia dalla venuta e dal messaggio di Gesù Cristo: e il tramite di questa analogia gli viene offerto dalla lettura del Quo Vadis? di Henryk Sienkiewicz. ${ }^{3}$ La citazione implicita rivela un atteggiamento di dissenso da vivere, si potrebbe dire, come testimonianza di una fede, da attestare e mettere alla prova del divenire storico con il proprio esempio e la propria dignità umana, per poi esserne plasticamente una prova e una dimostrazione, limpida ed evidente.

Il richiamo (il primo a uno scrittore polacco che si incontra in un testo di Sciascia) è qui usato forzatamente dal personaggio per sostenere lo sforzo di razionalizzare la propria illusione; ma Quo vadis? tornerà invece e ancora nella storia intellettuale dello scrittore, confermando con una precisa scelta lessicale l'interpretazione

3 E valga, a provare la coerenza intrinseca del ragionamento di Sciascia-Calogero, il seguente passo tratto dal romanzo, in cui l'apostolo Pietro si rivolge ai Romani: “«Voi seminate nel pianto e raccogliete nella gioia. Perché temete la potenza del male? Sulla terra, su Roma, sulle mura della città, sta il Signore che è in voi. Le pietre saranno bagnate di lacrime, la sabbia immersa nel sangue, le vallate piene dei vostri corpi, ma io dico che voi sarete vittoriosi. Il Signore si avanza alla conquista della città del delitto, dell'oppressione e dell'orgoglio, e voi ne siete le sue legioni. Egli ha redento col suo sangue e coi suoi spasimi i peccati del mondo; così egli vuole che voi redimiate col martirio e col sangue questo nido di nequizia. Questo egli annuncia per la mia bocca»" [Sienkiewicz 1915: 214-215]. 
qui proposta del dissenso da vivere come testimonianza. Nel 1982, infatti, il romanzo comparve nella collana "La memoria" della casa editrice Sellerio. Come spesso avveniva, Sciascia scrisse di proprio pugno (senza firmarsi), il risvolto di copertina. Ed eccone la prima parte, molto significativa:

Questo libro, che ebbe intensa e lunga popolarità fin dal suo apparire (1894-96: e la prima traduzione italiana - di Federico Verdinois - è del 1899) [...] si ristampa oggi per una duplice sollecitazione. È un libro cristiano, di un cristianesimo vissuto, di un cristianesimo «testimoniato», di un cristianesimo non facilmente e comodamente professato. Ed è un libro polacco, di una Polonia oggi in condizioni non molto diverse di quelle in cui si trovava al momento in cui Sienkiewicz lo concepiva e scriveva. È un libro - cristiano e polacco - di pace, di libertà [... $]$. È il libro di una causa - polacca e umana - ancora oggi terribilmente attuale [Nigro 2003: 110].

La pubblicazione del romanzo aveva dunque una duplice funzione, per lo scrittore (che dal portato etico del cristianesimo delle origini si sentiva profondamente coinvolto): ${ }^{4}$ ripubblicare Quo vadis? gli permetteva di proseguire la sua inquieta e personale ricerca di fede ("la religione va vissuta giorno per giorno, in conflitto con noi stessi, e anche dolorosamente; non è passiva accettazione di una verità una volta per tutte rivelatasi e in cui credere soltanto attraverso atti di routine" [Sciascia 1979: 64]), e gli offriva l'occasione di gettare un ponte tra l'infelice vicenda umana e spirituale delle prime comunità cristiane e le forme di controllo, oppressione e censura messe in atto dal regime sovietico nei paesi del blocco comunista. cristianamente, va senz'altro ricordata questa dichiarazione: "Leggo i vangeli; e anche spesso. Ne tengo una copia in città, a Palermo, e l'altra nella casa di Racalmuto: a portata di mano. Non c'è quasi giorno che non li riprenda $[\ldots]$. [È] una regola, ormai. Qualcosa come dar corda all'orologio perché non lo si trovi fermo l'indomani" [Messori 1987: 20]. 
Una simile analogia storica, altrettanto luminosa e pertinente, era stata proposta (sin dal titolo) nel 1969, per un testo al quale Sciascia era particolarmente legato: ${ }^{5}$ Recitazione della controversia liparitana, dedicata ad A.D., dove le iniziali puntate, com'è noto, si riferiscono ad Alexander Dubček, il leader della Primavera di Praga (conclusasi con l'ingresso dei carri armati russi nella capitale cecoslovacca, nell'agosto 1968). Il giudizio sul presente politico europeo (in questo caso la fine dell'eterodossa esperienza praghese del "socialismo dal volto umano" e la destituzione di chi la guidò, poste in essere dall'ortodossia della chiesa comunista sovietica) veniva dunque proposto attraverso il filtro dell'analogia storica con un tentativo di conduzione razionale del governo della chiesa cattolica nella Sicilia di primo Settecento (tentativo operato da un gruppo di giudici ed ecclesiastici dissenzienti che spesso, nel testo, si richiamano appunto ai valori del cristianesimo delle origini). ${ }^{6}$

Con la riproposta di Quo vadis?, Sciascia volle così richiamare l'attenzione dei lettori italiani sul tempo presente della popolazione polacca, in quel preciso e determinante momento storico. Nella visione dello scrittore siciliano, la Polonia del 1896 e la Polonia del 1982 si saldavano in un unico esempio di identità negata e di oppressione (per opera dell'occupazione imperiale russa, prima, e del controllo sovietico, in seguito). ${ }^{7}$ Allo stesso tempo, lo

"La commedia dovevo scriverla, era una trama che mi ossessionava" [Lombardo 2008: 101]: così scriveva Sciascia all'agente letterario Erich Linder nel settembre 1969.

6 Quanto Sciascia tenesse a quella dedica, e ai significati ad essa sottesi, si può rilevare da un altro passo della lettera già citata al suo agente letterario Linder: “[... $]$ mi pare di aver trovato un buon titolo: Recitazione della controversia liparitana, dedicata ad A.D. È sufficientemente incomprensibile, ma spero che lettori e spettatori capiscano che è dedicata a Dubček" [Lombardo 2008: 108]. Anche la lettera di accompagnamento delle bozze definitive, inviate alla casa editrice Einaudi, ne conferma la fondamentale importanza: "Ti raccomando il titolo: "dedicata ad A.D." ne fa parte, e in copertina e sul frontespizio va con lo stesso corpo della Recitazione della controversia liparitana. Assolutamente” [Sciascia 2012: 1982].

7 È il caso di notare che appena l'anno prima, nel maggio 1981, sulla rivista polacca "Literatura na Świecie" era apparsa la traduzione de La morte di Stalin: a quell'altezza le proteste e le manifestazioni popolari guidate dal sindacato libero Solidarność raccoglievano ormai milioni di lavoratori, e il racconto dell'illusione del 
scrittore-editore confermava la sua più che compiuta liberazione dal giogo dell'ideologia, che lo aveva portato per tempo ad assumere posizioni sempre più ferme e indipendenti, ispirate da un onesto e doveroso pensiero divergente.

\section{La contraddizione illuminista: Sciascia tra Brandys e Jerzy Lec}

Con La morte di Stalin e la creazione di un piccolo antieroe illuso e sconfitto dalla Storia, Sciascia marcava dunque il proprio distacco dalle ideologie, ${ }^{8}$ con segni di determinata consapevolezza e ironica leggerezza: cifre di un dissenso che lo scrittore non declinò mai nel senso estremo di un'opposizione assoluta, indifferenziata, respingente; piuttosto nell'accezione di una intatta e cosciente libertà del giudizio critico, da esercitare con indipendenza e assertività nella scrittura come nell'impegno politico.

Era un'impostazione affine ma non pienamente assimilabile a uno scrittore come Kazimierz Brandys, che incarnò il dissenso nelle sue forme più plastiche e tangibili dell'esilio volontario e dell'opposizione intellettuale, dopo una vicenda personale certo più dolorosa, lenta e accidentata di quella di Sciascia. ${ }^{9}$ Dopo il rapporto Chruščëv del 1956 e la denuncia dei crimini staliniani, la fede di Brandys nel comunismo non vacillò, anche se lo scrittore cominciò a recepire le distanze crescenti che separavano gli ideali dalle ottuse e soffocanti burocrazie di regime: finché, nel 1966, non decise di uscire dal PZPR (il Partito Operaio Unificato Polacco).

Se La morte di Stalin (pubblicato nel 1958, ma già pronto nell'estate del 1956 [cfr. Sciascia 2012: 1721]) è la cronaca della reazione

ciabattino Calogero offriva un emblematico exemplum a quanti ancora esitavano sulle posizioni da assumere [cfr. Schembari 2017].

8 Già esplicitato in un passaggio dell'introduzione a Le parrocchie di Regalpetra: "Con queste pagine non metto una bandiera rossa al pianterreno: non saprei goderne l'effetto dalla terrazza; né, restando al pianterreno, potrei salutarla con fede" [Sciascia 2014: 13].

9 Ma vale la pena di menzionare un'affermazione di intenti di Sciascia, raccolta dalla giornalista Julia Dobrovolskaja, e decisamente in sintonia con le scelte di tanti scrittori dell'Est europeo in dissenso con il regime sovietico: " $«$ Se dovessi trovarmi in uno stato di non libertà, emigrerei!». Fu la prima e ultima volta che sentii un italiano pronunciare una frase simile" [Dobrovolskaja 2006: 217]. 
disorientata ma ancora tenacemente illusa dei comunisti italiani seguita alla divulgazione del rapporto, il romanzo Matka Królów (La madre dei Re) certifica il sorgere dei primi segnali di incertezza da parte dei comunisti polacchi post-staliniani "tra il marzo 1956 e il marzo 1957, dodici mesi nei quali girò, insieme al sole, il modo dei comunisti di considerare sé stessi” [Rossanda 1987: 225]: un'incertezza non ancora ideologica, ma figlia di un'insofferenza crescente alle dinamiche sociali dettate dall'ideologia.

Soltanto venticinque anni dopo, sorpreso all'estero dalla legge marziale decretata il 13 dicembre 1981 dal generale Jaruzelski, ${ }^{10}$ Brandys scelse la via dell'esilio, stabilendosi definitivamente a Parigi: quando, però, era già ben altro scrittore rispetto a quello de La madre dei Re. Ormai libero dal giogo estetico e ideologico del realismo socialista (di cui era stato efficace e impegnato interprete), riversò la trepidazione e l'inquietudine degli anni che precedettero e seguirono l'esilio in una scrittura più intima, introspettiva, che univa cronaca, memoria e invenzione. Questa svolta stilistica confluì nella stesura di opere proteiformi, sospese tra narrativa e saggistica, fino ai Miesiace (Mesi), i diari scritti e pubblicati tra il 1978 e il 1987, testi dallo stile eclettico che lo scrittore giustificava con queste parole:

Alcuni critici sostengono che i miei libri sembrano scritti da tanti Brandys diversi. Ma io mi chiedo: come è possibile pretendere coesione e unitarietà d'intenti in un secolo come il nostro, che ha vissuto quello che ha vissuto? Il terrore fascista e bolscevico? Le guerre mondiali e l'Olocausto? Questi terremoti hanno distrutto ogni possibilità di una visione uni-

Sulla posizione di Sciascia riguardo al golpe in Polonia, oltre all'Intervista impossibile al generale Jaruzelski [Sciascia 2012: 1622-1624], si segnala l'articolo Quei polacchi, peggio per loro, comparso il 20 dicembre 1981 sulla "Gazzetta del Mezzogiorno", in cui lo scrittore dissente energicamente da chi accusava i lavoratori polacchi di non avere saputo avanzare le loro pur giuste proposte con gradualità e prudenza: "Si ignora così $[\ldots]$ che un processo di liberazione è totale e inarrestabile e che tirato il filo alla prima smagliatura non si può che tirarlo tutto a dissolvere tutta la tessitura, anche a costo di restarne strangolati” [Giacovazzo 2001: 95]. 
voca e coerente dell'umanità. Non sappiamo nemmeno più se apparentarla a un martire, a un villanzone, o a un genio. Stando così le cose, è ovvio che vada gambe all'aria anche il punto di vista dell'osservatore, dello scrittore... in cui ora albergano, costrette alla convivenza, le sensazioni più diverse e incompatibili: impotenza, pathos, orrore [Marcoaldi 2000].

L'incessante verifica del proprio giudizio etico e critico, anche a costo di contraddirsi, ${ }^{11}$ e la conseguente commistione dei generi, di adattamento della forma del testo alle esigenze espressive, sono chiari punti di contatto tra i due scrittori. ${ }^{12}$ Sono anche questi gli elementi che sostengono l'interferenza più nota di un testo di Brandys in Sciascia, la citazione dalle Listy do pani Z. (Lettere alla signora Z.) in A ciascuno il suo. È il passo in cui il professore-investigatore Laurana coinvolge ingenuamente l'avvocato Rosello, mandante dell'omicidio del dottor Roscio, e lo mette a parte dei suoi progressi nelle indagini. Dopo una di queste conversazioni, Laurana è invitato da Rosello a fare con lui un sopralluogo a casa

Ovvero, nelle parole di Sciascia: “[ $[.$.$] un uomo vivo ha diritto alla contraddi-$ zione. Mi piacerebbe anzi che l'epigrafe sulla mia vita fosse semplicemente questa «Contraddisse e si contraddisse». Una contraddizione, appunto, in nome della vita, in nome della speranza" [Sciascia 1982: 177].

Le possibili intersezioni tra i due scrittori non si esauriscono qui. Su tutte va almeno segnalata l'evidente fascinazione per l'autorevole modello culturale, etico ed estetico della "conversazione". Brandys intitolò uno dei suoi ultimi lavori Sztuka konwersacji (L'arte della conversazione), una raccolta di dialoghi già adattati e messi in scena a teatro due anni prima: sono racconti di re-incontri tra persone legate da vincoli ormai sciolti o indeboliti (ora un padre e una figlia divisi da tempo, ora due ex amanti), un "omaggio a qualcosa che scompare" [Rasy 1995: 4]. Il riferimento è a quella stessa civiltà della conversazione di cui Sciascia registrò, in numerosi punti della sua opera, l'inesorabile disgregazione lungo la storia della socialità europea dal Quattrocento al Novecento [cfr. Schembari 2015]; una perdita cui si poteva porre rimedio - per lo scrittore siciliano - recuperando quella tradizione come proficuo modello di scrittura, come civiltà dello "scrivere conversando" [cfr. Sciascia 2000, 37-43]. Di questa nuova civiltà egli stesso - "narratore «impuro»" [Sciascia 2012: 1796] per autodefinizione - si sentiva parte, in compagnia di alcuni dei suoi prediletti auctores, capaci di praticare una necessaria osmosi dei generi letterari: da Montaigne a Stendhal, da Borges a Savinio e - si può ben dire - allo stesso Brandys che "non ama i generi [...] allo stato puro, così che ne vi aderisce, né però li abbandona” [Rasy 1995: 3]. 
della vittima, dove, ad attenderli, c'è ovviamente la vedova Roscio, che li accoglie con l'ansia (ben simulata) di chi attende di conoscere nuovi dettagli sulla morte del marito:

Li guidò nello studio, una stanza un po' cupa o che così pareva per la luce che, cadendo sulla scrivania, lasciava in ombra gli scaffali severi, fitti di libri. Sulla scrivania era un libro aperto. «Lo stava leggendo» disse la signora. Tenendovi due dita in mezzo, a segnale, Rosello lo chiuse, lesse il titolo: «Lettere alla signora $Z$... che roba è? » domandò a Laurana. «Molto interessante, di un polacco» [...]. Laurana si chinò sul libro aperto, gli saltò all'occhio una frase: «Solo l'atto che tocca l'ordinamento di un sistema pone l'uomo nella cruda luce delle leggi $>$ e allargando la visione della pagina, quasi aprendo un diaframma e non scorrendo le righe, riconobbe il luogo del discorso, il contesto: dove lo scrittore parla di Camus, dello Straniero. 'L'ordinamento di un sistema! E dov'è qui il sistema? C'è mai stato, ci sarà mai? Essere stranieri, nella verità o nella colpa, e insieme nella verità e nella colpa, è un lusso che ci si può permettere quando c'è l'ordinamento di un sistema. A meno che non si voglia considerare sistema quello in cui il povero Roscio è scomparso. E allora l'uomo è più straniero nella parte del boia che in quella del condannato; più nella verità se manovra la ghigliottina, e meno se ci sta sotto' [Sciascia 2012: 556-557].

Il riferimento non è di facile interpretazione. Se l'infrazione di una norma dell'ordinamento "sembra la via maestra per certificare l'irriducibile individualità morale del singolo", per Laurana il sistema si scardina nel milieu sociale in cui Roscio ed egli stesso si trovano a vivere e poi trovano la morte, "dove, latitando lo Stato, l'uomo sembra poter esistere eticamente, nella verità o nella colpa, solo entrando in rapporto con un Potere, declinato dal lato della violenza arbitraria e non del delitto, che si autolegittima ed autoriproduce" [Onofri 2004: 134-135].

In questa luce, il passo interpolato proietta la vicenda di Laurana in una prospettiva di consapevolezza etica ed esistenziale complessa e stratificata, sofferta e irrisolta, che sarà poi quella 
degli investigatori di Sciascia che verranno, dall'ispettore Rogas de Il contesto al Vice de Il cavaliere e la morte. Il richiamo a quella pagina di Brandys, però, invita anche ad un'altra riflessione, che avvicina sensibilmente le esperienze e i pensieri dei due scrittori lungo le loro esistenze. Essa è tratta da una parte delle Lettere alla signora Z. che l'autore dedica alla responsabilità e all'autorità degli scrittori nella società:

Oggi scrivo per una ventina di amici; i miei libri è come se cadessero in un pozzo, non so chi li legge. Ma non sono capace di scrivere altro che non sia quel che ho da dire $[. .$.$] . Queste$ e simili confessioni potrà sentirle oggi da più di uno scrittore che, invece di cedere ragionevolmente di fronte al bisogno delle masse, si ostina a somministrare giustizia al mondo visibile [... . Ma il tempo dello scrittore ha questa particolarità: in lui tutto dura contemporaneamente e di tutte le cose passate egli crea un presente continuo. Da questo dipendono la sua forza e la sua deficienza; in questo consiste la sua morale [Brandys 2000: 110-112, passim].

Si scorge, in queste affermazioni, il riflesso di una vicenda intellettuale che si votava all'esercizio continuo del dubbio e al racconto della verità, come fu anche per Sciascia; ${ }^{13}$ e che accettava la condizione assegnata da Voltaire all'uomo di lettere nel suo Dictionnaire philosophique (in una pagina ben nota a Sciascia [cfr. Sciascia 1982: 38-40]): il destino, cioè, di vivere come il pesce volante che "s'innalza un poco, ed ecco che gli uccelli lo divorano; s'immerge, ed ecco che i pesci lo mangiano" [Voltaire 2013: 2193]. La citazione non sembri esornativa: la pagina volterriana da cui è tratta è evocata in un articolo di Sciascia per il settimanale "Epoca" del 12 aprile 1978, poi confluito - con poche modifiche - ne La palma va a nord; è una nota sul senso della parola "libertà", che muove dal tentativo di definire cosa significhi essere - in ogni tempo - illuministi: per una delle sue ultime opere pubblicate, la raccolta di interventi giornalistici su mafia e giustizia A futura memoria: "Preferisco perdere dei lettori, piuttosto che ingannarli” [Sciascia 2019: 1189]. 
L'uomo dei lumi $[\ldots]$ nel momento in cui propugna il diritto di opporsi a qualsivoglia autorità acquisisce il senso della contraddizione. Da quel momento può anche succedere che si trovi in contraddizione con sé stesso: egli diviene, allora, il primo critico delle idee dalle quali è attratto e delle formule che ama, fino al punto di volere tentare l'esperienza del contrario. Sotto questa definizione possiamo collocare tutti gli intellettuali: tutti coloro, cioè, che hanno la capacità, i mezzi e il tempo per tener desta la propria intelligenza. Cosa che comporta non il registrare passivamente, ma piuttosto il criticare in forma attiva. Vale a dire: tutti gli intellettuali sono stati, o sono uomini dei lumi [Sciascia 1982: 39].

La definizione, tolta e riformulata da L'invenzione della libertà di Jean Starobinski, delinea nella sua complessità una categoria critica cui si è fatto spesso ricorso (a rischio di inflazionarla) negli studi critici sullo scrittore; ma è la chiave per decifrare la comune matrice etica su cui Sciascia fondava, in effetti, la sintonia che avvertiva nei confronti di Brandys. È probabile che il saggio di Starobinski gli fosse già noto quanto meno dalla sua prima pubblicazione italiana, del 1965 [Starobinski 1965], che è anche l'anno in cui in Italia vengono pubblicati i Myśli nieuczesane (Pensieri spettinati) di Stanisław Jerzy Lec; puntualmente segnalati da Sciascia su “L’Ora” di Palermo:

Mentre rivedo Morte dell'inquisitore, per la ristampa $[\ldots] \mathrm{mi}$ imbatto nei Pensieri spettinati di Stanislaw Jerzy Lec che l'editore Bompiani pubblica in deliziosa edizione col titolo, forse più pertinente, di Pensieri proibiti: e sono pensieri che ossessivamente gravitano sul tema dell'inquisizione; non quella cattolica e spagnola, ma quella laica che accompagnò il momento staliniano della realizzazione del comunismo [...]. L'inquisizione, dunque: nella Polonia degli anni di Stalin come nella Spagna di Filippo II (e di Franco). E vissuta dal di dentro: con la stessa lucida repugnanza di un Voltaire, ma dentro una condizione di insicurezza personale, di continuo rischio, che Voltaire appena conobbe. Che è già un fatto abbastanza significante: l'illuminista del XviıI secolo correva rischi minori dell'illu- 
minista del xx. Perché Lec è un illuminista: così come l'altro scrittore polacco da cui ci viene quel lucidissimo messaggio che sono le Lettere alla signora Z., Casimiro Brandys [Sciascia 1991: 66].

"Vissuta dal di dentro": è il discrimine necessario per porre nella giusta luce la facies illuminista della vicenda intellettuale di Brandys e Lec, ${ }^{14}$ assunta non come "volontà smitizzante che riduca tutto alla scabra evidenza del razionalismo" ma come "natura fondante di indagine aperta, laica e non clericale" [Castelli 2016: 119], che fa scaturire la reazione ai dogmi omologanti delle inquisizioni di ieri e di oggi da un primigenio e fondamentale dissidio verso se stessi e la propria corresponsabilità storica nella creazione di quei sistemi oppressivi. È da quel punto di svolta interiore che è possibile tracciare i confini di una terra condivisa dove - anche per Sciascia - si afferma la "centralità delle esperienze della contraddizione e dell'antitesi” [Castelli 2016: 125].

\section{La tentazione della disobbedienza: il Vice e il procuratore} Fedorovič

Tra altri ancora da indagare, ${ }^{15} c^{\prime}$ è un ultimo riferimento intertestuale che può essere utile ricordare, a conclusione delle note qui proposte. Una suggestione, in realtà, che arriva dal racconto lungo Odpocznij po biegu di Władysław Terlecki, scritto nel 1975, tradotto

Che non a caso Sciascia citerà nella introduzione alla ristampa congiunta del 1967 - per Laterza - di Morte dell'inquisitore e delle Parrocchie di Regalpetra [cfr. Sciascia 1987: 6].

15 L'elenco degli scrittori polacchi che hanno attirato l'attenzione di Sciascia comprende almeno Jan Potocki, conte e viaggiatore da grand tour, cui Sciascia dedicò una nota, ancora su "L'Ora" di Palermo, complice la riedizione, nel 1965, del Manuscrit trouvé à Saragosse [cfr. Sciascia 1991: 101-102]; Joseph Conrad, "uno scrittore in cui entri con diffidenza, ma che poi ti prende in un modo che vorresti continuare all'infinito" [Sciascia 1982: 101]; senza dimenticare Andrzej Kuśniewicz, di cui Sellerio pubblicò Il Re delle Due Sicilie e Lezione di lingua morta, per citare solo quelli apparsi per volontà di Sciascia [cfr. Nigro 2003: 87, 113], che proprio nel 1989, anno della sua scomparsa, si batté per l'assegnazione del premio Savarese allo scrittore polacco. 
e pubblicato da Sellerio nel 1983, nella collana "La memoria", con il titolo In fondo alla strada. Non c'è evidenza di un intervento in prima persona di Sciascia per la pubblicazione di questo testo, ma è difficile pensare che il progetto editoriale fosse sconosciuto allo scrittore. E dunque: In fondo alla strada è il racconto delle indagini di Ivan Fedorovič, procuratore di Pietroburgo chiamato a investigare - nel cuore della Polonia cattolica e zarista di fine Ottocento - su un omicidio subito rubricato come "passionale" dalle autorità, e sulla scomparsa di un tesoro in gioielli (crimini avvenuti entrambi in un convento). ${ }^{16}$

Il principale indiziato, frate Sykstus, è reo confesso, ma l'indagine è impervia, intrecciata com'è alla politica imperialista russa nell'ormai scomparso "Regno del Congresso" polacco, e si dipana lungo le tormentate giornate del procuratore: che è malato, preda di improvvisi e dolorosissimi attacchi che lo lasciano prostrato e che, tuttavia, non lo distolgono dall' inquieto procedere verso una verità giudiziale che sia anche verità esistenziale, al punto di scontrarsi non di rado con Spiridion Apollonovič, il presidente del tribunale che imbastirà il processo.

Gli interrogatori, i sopralluoghi, e le lunghe visite presso il medico che lo ha in cura diventano infatti occasioni - per lui, uomo di una laicità e razionalità persino dure e manichee, in certi tratti - di lunghe disquisizioni con l'interlocutore di turno, o di soliloqui mentali sulla possibilità e verità del giudizio umano, sul ruolo di casualità e causalità nello svolgersi delle azioni individuali; sofferte e articolate meditazioni sul diritto e il peso morale del "giudicare", che richiamano - a posteriori, s'intende - quelle che avevano coinvolto l'ispettore Rogas nel Contesto e il pittore-investigatore di Todo Modo:

Sono costantemente circondato da innumerevoli paragrafi, codicilli e disposizioni ufficiali. Ma faccia a faccia con un criminale avverto ogni volta l'insufficienza di quelle norme. Bastano assassinò nel 1910 il cugino Wacław nella propria cella del monastero di Jasna Góra, confessando poi di avere derubato il cenobio e profanato l'icona della Madonna di Częstochowa. 
ad incriminare un uomo, certo, ma divengono del tutto inutili appena cerco di comprenderlo [...]. [Terlecki 1983: 46].

Ma la vicenda umana e professionale del procuratore Fedorovič mostra più di un'assonanza con quella di uno degli ultimi personaggi creati da Sciascia, il Vice de Il cavaliere e la morte (che è anche, com'è noto, il più dolorosamente autobiografico): l'oscura e dolorosa malattia, come detto - con l'esperienza del dolore che entrambi associano e assimilano alla percezione dei colori [cfr. Sciascia 2012: 1132; e Terlecki 1983: 185] - e un'indagine che deve fare i conti, da un lato, con il clima insurrezionalista polacco e la volontà del potere centrale di sfruttare il delitto (forse addirittura pianificato dal governo) per il proprio tornaconto [cfr. Terlecki 1983: 39-40; 229-230]; dall'altro, con un sistema di potere occulto, legato alla politica dal filo doppio della dipendenza e della connivenza [cfr. Sciascia 2012: 1164-1166], i cui interessi comuni concorrono a indirizzare l'investigazione del Vice verso la falsa pista del redivivo terrorismo estremista. ${ }^{17}$

A istruttoria conclusa (che indirizza comunque il processo secondo le direttive ministeriali) Ivan Fedorovič ha ormai imparato a "familiarizzare col pensiero della fine" misurando la "distanza [... $]$ e anche l'ostilità" che lo separano già dal "teatro $[\ldots]$ vivo, colmo di mistero $[\ldots] \mathrm{ma}[\ldots]$ completamente estraneo" dell'esistenza [Terlecki 1983: 224-225, passim]; così come al Vice accade "pur nella dilagante pietà che sentiva" di chiedersi se non ci fosse nel suo atteggiamento di distacco "il rancore di stare per morire el'invidia per coloro che restavano" [Sciascia 2012: 1183]. Finché la fine arriva davvero, con destini opposti per due personaggi diversamente disobbedienti, ma ugualmente inquieti.

Non si possono non segnalare, inoltre, le disquisizioni che entrambi i personaggi intessono sulla figura del diavolo, giungendo a conclusioni assimilabili. Secondo il procuratore Fedorovič per molti "il diavolo portava ancora coda e zoccolo fesso" ma quello di Sykstus era "un diavolo che pensava. Niente a che vedere con l'idiota del popolino che se ne stava in cima ad uno steccato a scrutare attorno coi suoi occhi da gatto" [Terlecki 1983: 58-59, passim]; per il Vice - allo stesso modo - il diavolo caprino che poteva contemplare nella prediletta incisione di Dürer - cui si ispira il titolo del racconto - "era troppo orribilmente diavolo per esser credibile. Gagliardo alibi, nella vita degli uomini, tanto che si stava in quel momento tentando di fargli riprendere il vigore perduto" [Sciascia 2012: 1172]. 
Sul treno che deve riportarlo a Pietroburgo, il procuratore apprende del suicidio della moglie di un vetturino coinvolto nel caso, che aveva proclamato fino in fondo la propria innocenza ed estraneità; il dubbio atroce di essere stato strumento di un'ingiustizia lo prende così un'ultima volta:

[vide] il proprio riflesso sovrapporsi al volto dell'uomo che stava dall'altro lato. Non capì se aveva di fronte i suoi occhi, o se erano gli occhi di quello che si era messo ad agitare il cappello [... ]. - I Suoi occhi ... - mormorò, pensando alla presenza di colui che castigava gli innocenti, in base a una giustizia dalle inconcepibili leggi $[\ldots]$. Ebbe un sussulto. Un dolore acuto. Ecco che rientrava nel suo regno. Strinse i pugni, e fissò il nero bagliore che lo ghermiva [Terlecki 1983: 254].

Nella disperante grazia che avvolge la conclusione de Il cavaliere e la morte, il Vice percepirà invece nitidamente la giustezza delle proprie intuizioni, ma quel suo ultimo pensiero "nella fine del tempo di cui stava varcando la soglia $[\ldots]$ era già, eterno e ineffabile, il pensiero della mente in cui la sua si era sciolta" [Sciascia 2012: 1188]. Se le somiglianze che si sono tratteggiate non esplicitano certo un rapporto di discendenza dell' investigatore sciasciano dal procuratore di Terlecki, i due finali sono accomunati da un'evidente chiave di lettura: la narrazione della morte come ultimo atto di vita, il suo materializzarsi nello sguardo e nel pensiero ancora consapevoli dei due personaggi. Per Sciascia un'ultima antitesi, la contraddizione finale di un' indagine sul senso della vita, appresa anche grazie alla lezione degli amati scrittori polacchi.

\section{Bibliografia}

Biagi Enzo (1973), Dicono di lei: Sciascia, "La Stampa", 10 giugno, p. 3. Bosco Gabriella (1998), Sciascia e la Francia. Storia di un'appartenenza,

"Franco-Italica", n. XIII, pp. 219-232.

Brandys Kazimierz (1987), La madre dei Re, trad. di Ludovico Tulli, Feltrinelli, Milano. 
Brandys Kazimierz (1994), L'arte della conversazione, trad. di Raffaella Belletti, E/O, Roma.

Brandys Kazimierz (1983), Mesi, trad. di Giovanna Tomassucci, E/O, Roma.

Brandys Kazimierz (2000), Lettere alla signora Z., trad. di Franca Wars, La Vita Felice, Milano.

Castelli Rosario (2016), Contraddisse e si contraddisse. Le solitudini di Leonardo Sciascia, Franco Cesati Editore, Firenze.

Della Terza Dante (1990), Sciascia e la Francia, in Omaggio a Leonardo Sciascia, atti del convegno di Agrigento, 6-7-8 aprile 1990, Provincia Regionale di Agrigento, Agrigento, pp. 199-212.

Dobrovolskaja Julia (2006), Post scriptum. Memorie. O quasi, Libreria Editrice Cafoscarina, Venezia.

Drago [i.e. Kalajić Dragoš] (1964), Warzecha, L’Obelisco, Roma. Ferraro Bruno (1993), Una citazione di Kazymierz Brandys in "A ciascuno il suo di Leonardo Sciascia", "Il lettore di provincia", n. 88, xxv, pp. 19 -29.

Fontana Albertina, Pupo Ivan (2019) (a cura di), Nel paese di Cunegonda. Leonardo Sciascia e le culture di lingua tedesca, Leo Olschki Editore, Firenze.

Giacovazzo Giuseppe (2001) (a cura di), Sciascia in Puglia, Edisud, Bari. Gioviale Fernando (2000), La meravigliosa giustizia naturale. Verso Cervantes, con Erasmo e Pirandello, in: Id., Scenari del racconto. Mutazioni di scrittura nell'Otto-Novecento, Salvatore Sciascia, Caltanissetta-Roma, pp. 240-251.

Manganaro Paolo (1990), Sciascia e la Spagna, in: Omaggio a Leonardo Sciascia, atti del convegno di Agrigento, 6-7-8 aprile 1990, Provincia Regionale di Agrigento, Agrigento, pp. 191-198.

Kazana Leszek (1997), Scrittori polacchi in Sciascia: Kazimierz Brandys, in: La morte come pena in Leonardo Sciascia. Da «Porte aperte» all'abolizione della pena di morte, a cura di Italo Mereu, La Vita Felice, Milano, pp. 141-172.

Lombardo Giovanna (2008), Il critico collaterale. Leonardo Sciascia e i suoi editori, La Vita Felice, Milano.

Marcoaldi Franco (200o), Kazimierz Brandys, molti scrittori in uno, "Repubblica", 15 marzo.

Martinoni Renato (2011) (a cura di), Troppo poco pazzi. Leonardo Sciascia nella libera e laica Svizzera, Leo Olschki Editore, Firenze. Messori Vittorio (1987), Inchiesta sul cristianesimo, Società Editrice Internazionale, Torino. 
Morrison Ian (2009), Leonardo Sciascia's French authors, Peter Lang, Bern.

Nigro Salvatore Silvano (2003) (a cura di), Leonardo Sciascia scrittore editore ovvero La felicità di far libri, Sellerio, Palermo.

Nocera Antonina (2020), Metafisica del sottosuolo, Divergenze, Pavia.

Onofri Massimo (2004), Storia di Sciascia, Laterza, Roma-Bari.

Rasy Elisabetta (1995), Brandys, felici i pochi che sanno conversare,

"La Stampa-Tuttolibri”, 17 giugno, pp. 3-4.

Ricorda Ricciarda (1977), Sciascia ovvero la retorica della citazione, "Studi novecenteschi”, n. vi, pp. 59-93.

Ricorda Ricciarda (2015) (a cura di), Leonardo Sciascia e la Jugoslavia, Leo Olschki Editore, Firenze.

Rossanda Rossana (1987), Dodici mesi, in: Kazimierz Brandys, La madre dei Re, trad. di Ludovico Tulli, Feltrinelli, Milano, pp. 225-237.

Schembari Andrea (2015), "Causeries" siciliane. Leonardo Sciascia e l'etica della conversazione, in: L'Italia e la cultura europea, a cura di Anna Klimkiewicz, Maria Malinowska, Alicja Paleta, Magdalena Wrana, Franco Cesati Editore, Firenze, pp. 141-148.

Schembari Andrea (2017), «Dove il diavolo dà la buonanotte».

La ricezione di Leonardo Sciascia in Polonia, fra tentazione del cliché e primato dell'etica, "Todomodo", n. 7, pp. 319-334.

Sciascia Leonardo (1979), La Sicilia come metafora, intervista di Marcelle Padovani, Mondadori, Milano.

Sciascia Leonardo (1982), La palma va a nord, a cura di Valter Vecellio, Gammalibri, Milano.

Sciascia Leonardo (1987), Opere 1956-1971, a cura di Claude Ambroise, Bompiani, Milano.

Sciascia Leonardo (1991), Quaderno, Nuova Editrice Meridionale, Palermo.

Sciascia Leonardo (2012), Opere, a cura di Paolo Squillacioti, vol. I, Adelphi, Milano.

Sciascia Leonardo (2014), Opere, a cura di Paolo Squillacioti, vol. II, tomo I, Adelphi, Milano.

Sciascia Leonardo (2019), Opere, a cura di Paolo Squillacioti, vol. II, tomo II, Adelphi, Milano.

Sienkiewicz Henryk (1915), Quo vadis? Narrazione del tempo di Nerone, trad. di Paolo Valera, Sonzogno, Milano.

Simonetta Marcello (1996) (a cura di), Non faccio niente senza gioia. Leonardo Sciascia e la cultura francese, La Vita Felice, Milano.

Tedesco Natale (1989), Introduzione, in: L. Sciascia, Ore di Spagna, Pungitopo, Patti (Me). 
Tedesco Natale (1991), L'influsso spagnolo e la classicità di Sciascia, in: Id., La scala a chiocciola: scrittura novecentesca in Sicilia, Palermo, Sellerio, pp. 92-96.

Terlecki Ryszard (1983), In fondo alla strada, trad. di Alberto Zoina, Sellerio, Palermo.

Voltaire (2013), Dizionario filosofico, Bompiani, Milano.

Warzecha Marian (2018), [Intervista per il Muzeum Sztuki Wspótczesnej $w$ Krakowie], [consultato: il 26 giugno 2020], https://tinyurl. com/2wf952ee.

Andrea Schembari

\section{Masters of Dissent: Leonardo Sciascia and the Lesson of Polish Writers}

The article focuses on the intertextual presence of Polish writers in Leonardo Sciascia's work. Highlighted in this context is the possible influence on the Sicilian writer of the peculiar expression of "dissent" by writers such as Sienkiewicz, Brandys and Lec. The article closes with a brief comparison between the protagonists of Il cavaliere e la morte by Sciascia and Odpocznij po biegu (Rest after run) by Terlecki, both restless investigators disobedient to the authorities.

Keywords: Leonardo Sciascia; Italian 2oth-century literature; Polish 19th-2oth-century literature: dissent; writers' social responsibility; disobedience; intertextuality.

Andrea Schembari - dottore di ricerca in Filologia Moderna (Università di Catania, 2011), dal 2019 è docente e ricercatore confermato presso l'Istituto di Letteratura e Nuovi Media dell'Università di Stettino, dove insegna Letteratura comparata e Letteratura italiana contemporanea. È redattore della rubrica Traduzioni di "Todomodo" - Rivista internazionale di studi sciasciani, pubblicata da Olschki. Sullo scrittore siciliano ha pubblicato articoli e curatele. Per la casa editrice dell'Università di Stettino è in uscita per il 2020 la monografia Liturgia della fede civile. La controversia liparitana dai riformatori del "buon gusto" a Leonardo Sciascia. Contatto: andrea.schembari@usz.edu.pl. 
